\title{
The 2018 Revision of the ISBER Best Practices: Summary of Changes and the Editorial Team's Development Process
}

\author{
Lori D. Campbell, Jonas J. Astrin, ${ }^{2}$ Yvonne DeSouza, ${ }^{3}$ Judith Giri, ${ }^{4}$ Ashokkumar A. Patel, ${ }^{5}$ \\ Melissa Rawley-Payne, ${ }^{6}$ Amanda Rush, ${ }^{7}$ and Nicole Sieffert ${ }^{8}$
}

\begin{abstract}
An increased need for specimens of reliable and consistent quality for research purposes requires the development of standardized policies and practices for the collection, handling, storage, retrieval, and distribution of specimens and specimen-related data. Providers of specimen resources should strive to incorporate new technologies and state-of-thescience approaches and thus ensure the availability of fit-for-purpose research specimens. Strategies to achieve quality outcomes and performance improvements often include adherence to established standards and implementation of best practices. Although standards represent a rigid set of guidelines that define exactly how a task should be completed, best practices are recommended actions and principles that demonstrate an awareness of standards, solve problems, can be replicated, and work in a given context. Adoption of best practice elements will vary based on the goals and circumstances of a given initiative, and in some instances, may not be possible to implement or may represent an aspirational achievement. In an effort to harmonize the scientific, technical, legal, and ethical issues relevant to repositories of biological and environmental specimens, the International Society for Biological and Environmental Repositories (ISBER) has released the updated ISBER Best Practices: Recommendations for Repositories (ISBER Best Practices). The document provides a comprehensive tool to guide repository professionals in both managerial and technical aspects such as practical details on repository governance, development, and operation; regulatory compliance; and ethical, legal, and social issues relevant to repositories. This summary describes the process for revising the document and summarizes the new topics, updates, and areas of expansion included in the fourth edition of ISBER Best Practices.
\end{abstract}

Keywords: operating procedures, repository management, best practices, biospecimen science, biobank management

\section{Steps Toward Harmonization and Standardization}

$\mathrm{K}$ EY OBJECTIVES OF THE International Society for Biological and Environmental Repositories (ISBER) are to share successful strategies on providing fit-for-purpose specimens for research and to develop harmonized principles in the science and management of repositories. ISBER Best Practices* are intended to serve as a target for the quality management of specimens and to provide guidance for those seeking to improve their policies and procedures. They provide a helpful starting point in the evaluation and optimization of existing practices, development of new procedures, and performance of administrative oversight. In addition, adoption of these recommendations may support organizations seeking accreditation or certification by entities that accredit a repository according to national or international standards. These practices will continue to

${ }_{2}^{1}$ Cryogenic Control, Duluth, Georgia.

${ }^{2}$ Zoologisches Forschungsmuseum A. Koenig (ZFMK), Bonn, Germany.

${ }^{3}$ University of California, San Francisco, California.

${ }^{4}$ Biorepository Consultant, Augusta, Georgia.

${ }_{5}^{5}$ AIDS and Cancer Specimen Resource, Biodata Solutions, Cleveland, Ohio.

${ }^{6}$ Celgene Corporation, Summit, New Jersey.

${ }^{7}$ Children's Cancer Research Unit, Kids Research Institute, The Children's Hospital at Westmead, Westmead, Australia.

${ }^{8}$ Biospecimen Research Office, MD Anderson Cancer Center, Houston, Texas.

*Editor's Note: The full document of the ISBER Best Practices: Recommendations for Repositories, Fourth Edition, can be downloaded for free from the ISBER website at isber.org/bestpractices

(C) Lori D. Campbell et al., 2018; Published by Mary Ann Liebert, Inc. This is an Open Access article distributed under the terms of the Creative Commons Attribution License, which permits unrestricted use, distribution, and reproduction in any medium, provided the original work is properly cited. 
evolve to reflect developments in biospecimen science, present technological innovations, and remain current in relevant ethical and legal policies and regulations. ISBER's continued engagement of stakeholders and experts in repository management is essential for the provision of accurate and effective practices in the collection, handling, storage, retrieval, and distribution of biological and environmental specimen resources.

\section{Establishment and Evolution of Best Practices for Repositories}

In 2005, ISBER published Best Practices for Repositories I: Collection, Storage, and Retrieval of Human Biological Materials for Research (ISBER Best Practices) as a comprehensive tool to guide repository professionals in the technical, legal, ethical, and/or managerial issues relevant to repositories of biological and environmental specimens. ISBER Best Practices were subsequently revised in 2008 to include considerations and policies for specimens of nonhuman origin. The third edition was revised in 2012 to reflect advances in biospecimen science and new technology platforms. Topics covered in ISBER Best Practices include repository development considerations, organizational requirements, and personnel; funding, cost management, and other financial issues; physical facility management aspects such as security, fire prevention, emergency preparedness, storage equipment, and environments; specimen packaging and shipping requirements, regulations, and tracking; quality management including standard operating procedures development and maintenance, quality standards, method validation, and audits; general safety issues and local, regional, and national regulations; training programs, documentation, and records; specimen collection considerations; legal and ethical issues for collection of biospecimens; and sharing and distribution of specimens and data.

Building on the foundation of the previous versions, the fourth edition of ISBER Best Practices addresses scientific, technical, legal, and ethical issues relevant to repositories of biological and environmental specimens. Recommendations provided in the document reflect the collective knowledge and experience of ISBER members in supplying specimens and associated data in a form that meets specific quality criteria and in compliance with all necessary requirements and regulations. Rather than providing detailed procedures, the document consists of principles by which such policies and procedures should be developed. It is understood that achieving full compliance with the practices established in the document is regarded as the gold standard of repository success, but it is recognized that it will not be feasible for all repositories to achieve this. Repositories should decide how best to incorporate these recommendations and should refer directly to their own national/federal, regional, and local regulations and requirements, as appropriate.

\section{Development Process for the Fourth Edition}

The process for development of the fourth edition of ISBER Best Practices began in November 2016 with an announcement to the ISBER membership-at-large, asking for expressions of interest to participate as an editor or contributor. An Editorial Board was selected from the list of volunteers and multiple teleconferences were conducted to discuss the document format, content additions and expansions, timeline for the project, and procedures for updating the document. Editors were assigned a section or group of related sections from the third edition of the ISBER Best Practices. Each editor led discussions with contributors and incorporated suggested edits into a working draft. In February 2017, editors submitted revised drafts and a summary of edits for their assigned section(s) to the Editorial Board for review and input. Review drafts were then prepared and submitted to the ISBER Standards Advisory Committee (SAC) and Best Practices' International Review Board in March 2017.

Feedback from the ISBER SAC and Best Practices' International Review Board was shared with section contributors and members of ISBER Working Groups and Special Interest Groups for consideration. Comments and edits for each section were incorporated into a single working draft for discussion by the Editorial Board. A review highlighting areas lacking consensus was prepared and presented to the ISBER SAC in September 2017. The Editorial Board also asked for guidance on standardized terminology and applicable definitions for usage throughout the document. Input from the ISBER SAC was then incorporated into a final draft and submitted to the ISBER Board of Directors in October 2017 for comments and approval.

\section{Revisions, Areas of Expansion, and New Topics}

The fourth edition of the ISBER Best Practices is a revision of the third edition published in 2012 with an aim to include new areas of interest to the biobanking community, expand and update existing topics, and present an international perspective that reflects the growing diversity of the ISBER membership. Table 1 contains a summary of changes between the third and fourth editions of the Best Practices.

Throughout the document, the term "Best Practice" is used in situations where a level of operation is indicated that is above the basic recommended practice or more specifically designates activities or methods that have been successfully used by multiple organizations but have not yet become common practice. Repositories facing challenges that make it difficult or impossible to follow the recommended "Best Practices" should decide how best to incorporate these recommendations to meet their specific needs and requirements. It is important to note that some aspects of specimen management are governed by local, national/ federal, or regional regulations, and repositories should refer directly to these regulations and requirements, as appropriate. In addition to providing guidance and effective processes, ISBER Best Practices focuses on the establishment of standardized terminology and definitions for use by the global biobanking community. An example of these efforts is a clarification in the document of the use of the terms "specimen" and "sample," which vary significantly when used in a clinical versus biodiversity and/or environmental setting.

\section{Community Involvement and International Perspective}

ISBER serves as an international resource for the development, management, and operations of repositories 
Table 1. Summary of Changes Between the Third and Fourth Editions of ISBER Best PRaCtices

Significant revisions $\begin{array}{ll}\text { Section updates } \\ \text { I } & \text { Informatics updates in Repository Information Management Systems } \\ \text { K5.1.1 Changes made to collection of Surgical Samples under Solid Tissues section of Specimen Collection, } \\ \text { Processing, Receiving, and Retrieval }\end{array}$

Section title and related content changes

C Storage Equipment and Environments retitled to Storage and Processing Equipment

I Biological Material Tracking retitled to Repository Information Management Systems

K Specimen Collection, Processing, and Retrieval retitled to Specimen Collection, Processing, Receiving, and Retrieval

M Specimen Access, Utilization, and Destruction retitled to Specimen Access, Utilization, and Disposition

M2.6 Publishing and Provision of Data to Repositories condensed and retitled to Acknowledging Repositories and Reporting Utilization

Section relocations

D Records Management incorporated into Quality Management

E Method Validation and Quality Control Considerations (previously part of Quality Management) separated into new section

E2.1 Assessment of Cell Viability moved from Specimen Collection, Processing, Receiving, and Retrieval to Method Validation and Quality Control Considerations

Areas of expansion

\begin{tabular}{|c|c|}
\hline A 3.5 & $\begin{array}{l}\text { Expansion of Establishing Repository Models to include Federated Collections and Biodiversity } \\
\text { and Environmental Biobanks }\end{array}$ \\
\hline A3.1 & Development of a repository business plan discussed in greater depth \\
\hline $\mathrm{D}, \mathrm{F}$ & Significant updates in areas addressing Safety and Quality Management \\
\hline K3.4 & $\begin{array}{l}\text { Additional details added to Freeze/Thaw and Cooling/Re-warming Cycles in Specimen Collection, } \\
\text { Processing, Receiving, and Retrieval }\end{array}$ \\
\hline $\mathrm{K}$ & $\begin{array}{l}\text { Additional specimen types and processes were added to Specimen Collection, Processing, } \\
\text { Receiving, and Retrieval }\end{array}$ \\
\hline $\mathrm{L}$ & $\begin{array}{l}\text { Significant expansions to Collection of Non-human Biospecimens in Legal and Ethical Issues } \\
\text { for Biospecimens }\end{array}$ \\
\hline M3 & $\begin{array}{l}\text { Specimen or Collection Disposition created to address Culling, Specimen Destruction, and Transfer } \\
\text { of a Collection }\end{array}$ \\
\hline
\end{tabular}

New topics

A Repository Planning Considerations now includes Repository Governance and Development of a Governance Plan

B9 Pest and Contamination Control added to Facilities

C1.2 Guidance on identification of appropriate specimen storage container labels/identifiers for the type of storage equipment used

C Automated Storage Systems, Storage of FFPE Tissue Blocks and Slides, and Automated Liquid Handling Robotics added to Storage and Processing Equipment

D4.3 Quality Assurance (Non-conformities, KPI, CAPA)

F6 Biological Hygiene Plan, Compressed Gasses added to Safety

I Cloud Computing, descriptions of Data Exchange Standards, and RFID tags added to Repository Information Management Systems

J5 Cold Chain and Cold Chain Validation added to Packaging and Shipping

L3.1 Added considerations for Access and Benefit Sharing of specimens of other than human origin

Section numbers refer to relevant section(s) in the fourth edition.

CAPA, corrective and preventive actions; FFPE, formalin-fixed paraffin-embedded; ISBER, International Society for Biological and Environmental Repositories; KPI, key performance indicators; RFID, radio-frequency identification.

representing more than 30 countries worldwide. Since it is impossible to incorporate the national/federal, regional, and local regulations that apply to each repository, ISBER Best Practices strive to address areas of commonality among all repositories. In a similar manner, the document utilizes terminology that is generally understood among all repositories, limiting the use of sector-specific terms and providing an expanded glossary for assistance. Where terms are used ambiguously in the biobanking community, the glossary (referenced throughout the document) provides clarification.

Members of the Editorial Board and International Review Board represent a variety of repositories focusing on biological and environmental materials that are based in locations around the world. In addition, contributions and feedback from the diverse ISBER membership were encouraged to prevent any local, national/federal, or regional biases. 


\section{Commitment to Future Needs}

ISBER is the leading global forum for the dissemination of state-of-the-science policies, processes, and research findings. It aims to foster collaborations, create education and training opportunities, and provide an international showcase for cutting-edge technologies, products, and services. The organization seeks to promote harmonized highquality standards and ethical principles that cut across the broad range of repositories that ISBER serves. These efforts are supported by the publication of best practices that continue to evolve to reflect developments in biospecimen research science; advances in scientific, technological, and laboratory practices; and emergence of new ethical and legal policies and regulations. This commitment to maintaining current and scientifically accurate practices is reinforced by continuing efforts to solicit input from stakeholders in the biological and environmental specimen repository community. The ISBER Best Practices will play a key role as a valuable reference for new and established repositories in evaluating their current processes and providing guidance in the development and implementation of standardized practices.

The full document can be downloaded at no cost from the ISBER website (www.isber.org/bestpractices). For inquiries or information on participation in the development of ISBER Best Practices, please contact: (info@isber.org).

\section{Author Disclosure Statement}

No conflicting financial interests exist.

Address correspondence to: Lori D. Campbell, PhD Cryogenic Control 1820 Noblin Ridge Way Duluth, GA 30097

E-mail: loricampbel18647@gmail.com 